\title{
Clioquinol-Zinc Chelate: A Candidate Causative Agent of Subacute Myelo-Optic Neuropathy
}

\author{
Jack L. Arbiser, ${ }^{1}$ Stine-Kathrein Kraeft, ${ }^{2}$ Robert van Leeuwen, ${ }^{3}$ \\ Selwyn J. Hurwitz, ${ }^{2}$ Martin Selig, ${ }^{4}$ G. R. Dickersin, ${ }^{4}$ \\ Alan Flint, ${ }^{5}$ H. Randolph Byers, ${ }^{6}$ and Lan Bo Chen ${ }^{2}$ \\ ${ }^{1}$ Department of Dermatology, Emory University School of Medicine, \\ Atlanta, Georgia, U.S.A. \\ ${ }^{2}$ Department of Cancer Biology, Dana-Farber Cancer Institute, Boston, \\ Massachusetts, U.S.A. \\ ${ }^{3}$ Department of Dermatology, University of Leiden, Leiden, The \\ Netherlands \\ ${ }^{4}$ Department of Pathology, Massachusetts General Hospital and \\ Harvard Medical School, Boston, Massachusetts, U.S.A. \\ ${ }^{5}$ Department of Genetics, Children's Hospital, Boston, Massachusetts, U.S.A. \\ ${ }^{6}$ Department of Dermatology, Boston University School of Medicine, \\ Boston, Massachusetts, U.S.A.
}

Communicated by J. Folkman. Accepted August 1, 1998.

\begin{abstract}
Background: 5-chloro-7-iodo-8-hydroxyquinoline (clioquinol) was used clinically three decades ago as an oral antiparasitic agent and to increase intestinal absorption of zinc in patients with acrodermatitis enteropathica, a genetic disorder of zinc absorption. Use of clioquinol was epidemiologically linked to subacute myelo-optic neuropathy (SMON), characterized by peripheral neuropathy and blindness, which affected 10,000 patients in Japan. Discontinuation of oral clioquinol use led to elimination of SMON, however, the mechanism of how clioquinol induces neurotoxicity is unclear.

Materials and Methods: We tested the effect of clioquinol-metal chelates on neural crest-derived melanoma cells. The effect of clioquinol chelates on cells was further studied by electron microscopy and by a mitochondrial potential-sensitive fluorescent dye.
\end{abstract}

Results: Of the ions tested, only clioquinol-zinc chelate demonstrated cytotoxicity. The cytotoxicity of clioquinolzinc chelate was extremely rapid, suggesting that its primary effect was on the mitochondria. Electron microscopic analysis demonstrated that clioquinol-zinc chelate caused mitochondrial damage. This finding was further confirmed by the observation that clioquinol-zinc chelate caused a decrease in mitochondrial membrane potential.

Conclusions: We demonstrate that clioquinol, in the presence of zinc, is converted to a potent mitochondrial toxin. The phenomenon of clioquinol mediated toxicity appears to be specific to zinc and is not seen with other metals tested. Since clioquinol has been shown to cause increased systemic absorption of zinc in humans, it is likely that clioquinol-zinc chelate was present in appreciable levels in patients with SMON and may be the ultimate causative toxin of SMON.
Address correspondence and reprint requests to: Dr. Jack L. Arbiser, Department of Dermatology, Emory University School of Medicine, Woodruff Memorial Building, Rm. 5309, Atlanta, GA 30322, U.S.A. Phone: (404) 727-5872; Fax: (404) 727-5878; E-mail: jarbise@emory.edu

\section{Introduction}

5-chloro-7-iodo-8-hydroxyquinoline (clioquinol) has been used orally as an antiparasitic and in the treatment of acrodermatitis enteropathica, 


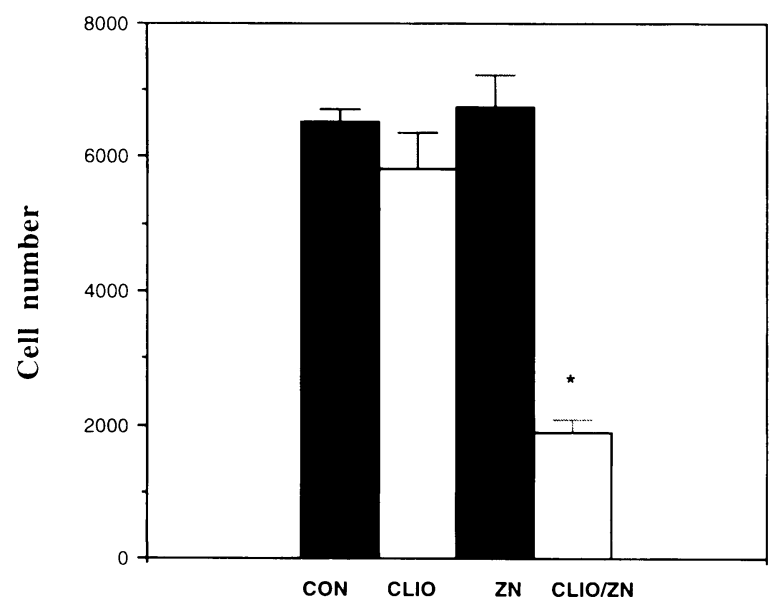

Fig. 1. Cell survival curve of MMAN cells after 24 hr of treatment with clioquinol, zinc, combination therapy, or vehicle alone. The number of cells in the clioquinol-plus-zinc treatment is significantly different from the values in the other three columns. The error bars represent SEM. The asterisk represents $p<0.05$.

an inherited inability to absorb zinc (1-5). Although clioquinol was effective in the treatment of acrodermatitis enteropathica, oral use of clioquinol was discontinued as it has been associated with subacute myelo-optic neuropathy (SMON), a condition characterized by peripheral neuropathy and blindness. Over 10,000 patients in Japan were affected by SMON before clioquinol was discontinued (2). The mechanism of how clioquinol causes SMON is currently unknown. Multiple theories have been ascribed, including synergistic toxicity with pesticides and viral infection, but none have been conclusively proven $(6,7)$.

In order to determine the mechanism of clioquinol toxicity, chelates of clioquinol with common ions were prepared, and cytotoxicity was tested on neural crest-derived melanoma cells. Of the metals tested, only clioquinol-zinc chelate showed toxicity towards melanoma cells. Zinc chelates with other agents, such as penicillamine and 1,10-phenanthroline, showed no cytotoxicity. The rapid course of cytotoxicity seen in the presence of clioquinolzinc chelate suggested that the target of toxicity was the mitochondria. Using mitochondrial-specific dyes, clioquinol-zinc chelate was found to cause a rapid decrease in mitochondrial membrane potential. Because clioquinol has been shown to cause increased systemic levels of zinc in humans due to formation of clioquinol-zinc chelate, and clioquinol-zinc chelate is a mitochondrial toxin, clioquinolzinc chelate is a likely cause of SMON.

\section{Materials and Methods}

\section{Cell Culture}

MMAN is a human melanoma cell line originating in this laboratory, originally derived from a lymph node metastasis (8). MMAN cells were maintained in Dulbeccos modified Eagle medium (DMEM; Sigma Chemical Co., St. Louis, MO) supplemented with $10 \%$ fetal calf serum (Hyclone, Logan, UT), penicillin, and streptomycin. Cells were subcultured weekly at a ratio of $1: 4$. A stock solution of $1 \mathrm{mg} / \mathrm{ml} \mathrm{5-chloro-7-iodo-8-hy-}$ droxyquinoline (Sigma) was prepared by reconstituting the drug in absolute ethanol or dimethylsulfoxide. Cells were grown in DMEM supplemented with $5 \%$ fetal calf serum. Zinc chloride, calcium chloride, magnesium sulfate, and copper sulfate were reconstituted as a $50-\mathrm{mM}$ stock solution in water and filter sterilized prior to use. Solutions of clioquinol and aqueous ion solutions were mixed together prior to addition of media to allow for optimal chelate formation.

\section{Cell Survival Assay}

Ten thousand MMAN cells were plated in 24well dishes. Twenty-four hours after plating, media were replaced with media containing clioquinol alone at a concentration of $7.5 \mu \mathrm{g} / \mathrm{ml}$, zinc chloride at $50 \mu \mathrm{M}$, the combination of both drugs, or vehicle alone (control cells). After $24 \mathrm{hr}$ of treatment, cells were counted using a Coulter counter (Hialeah, FL). Each experimental treatment was performed in triplicate.

\section{Electron Microscopy}

Cells for electron microscopic studies were grown in 35-mm plastic petri dishes and treated with test compounds for $24 \mathrm{hr}$. All steps were performed at room temperature unless otherwise noted. The cells were fixed for $45 \mathrm{~min}$ in KII fixative $(2.5 \%$ glutaraldehyde, $2 \%$ formaldehyde, $0.025 \% \mathrm{CaCl}_{2}$ in a $0.1 \mathrm{M}$ cacodylate buffer, $\mathrm{pH}$ 7.4) and washed in buffer. The cells were then post-fixed with $1.2 \%$ osmium tetroxide in collidine buffer for $\mathrm{l} \mathrm{hr}$, dehydrated in graded ethanol solutions, stained en bloc with $2 \%$ uranyl acetate, infiltrated with ethanol/epon mix- 


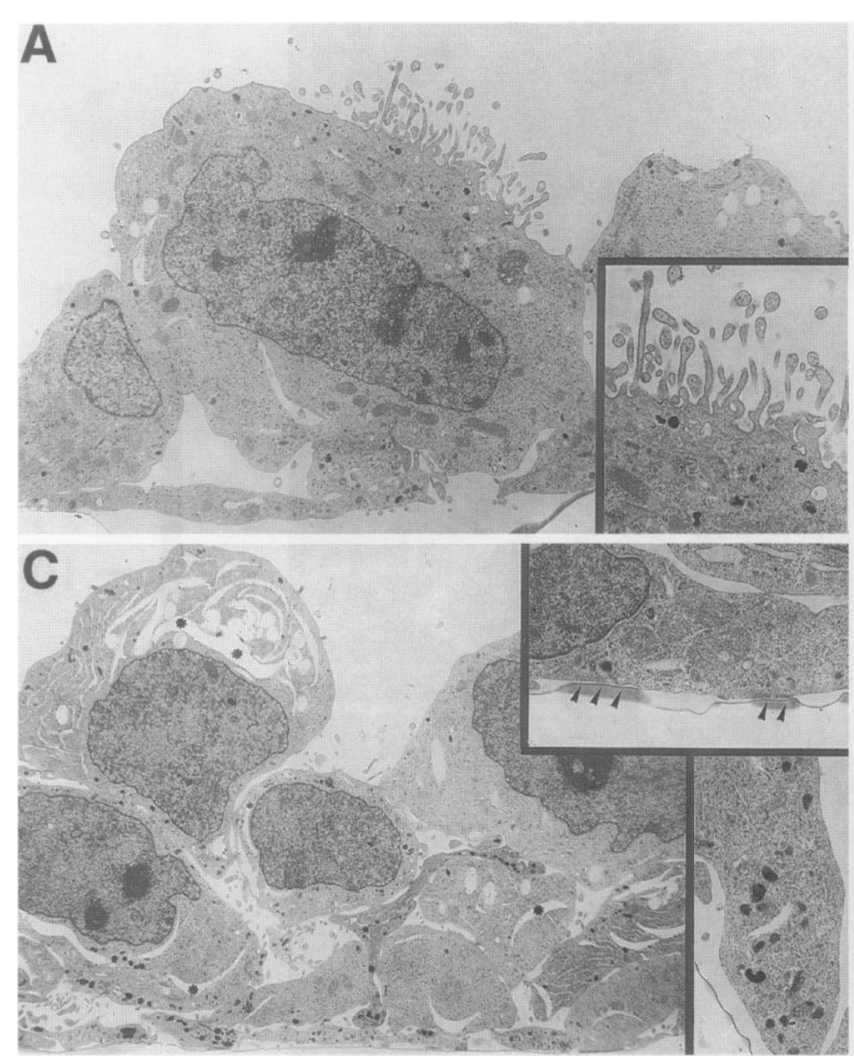

Fig. 2. Electron microscopy of MMAN cells treated with clioquinol and zinc. (A) Control melanoma cells $(\times 5800)$. The inset demonstrates microvilli present in the control melanoma cells $(\times 12,000)$. (B) Melanoma cells following treatment with $150 \mu \mathrm{M}$ zinc chloride $(\times 6000)$. (C) Melanoma cells following treatment with $7.5 \mu \mathrm{g} / \mathrm{ml}$ clioquinol. The asterisks in the central photograph $(\times 5300)$ indicate dilated endoplasmic reticulum. The arrows in

tures, and embedded in 100\% epon. Representative sections were stained with Sato's lead and examined with a Phillips 301 electron microscope at $40 \mathrm{kV}(9)$.

\section{Mitochondrial Membrane Potential}

Prior to measurements, $5 \times 10^{4}$ cells were plated in 8-well coverglass tissue culture chambers (Nunc Inc., Naperville, IL). Cells were loaded with the potential-sensitive dye MitoTracker Red CMXRos (50 nM, Molecular Probes, Eugene, OR) in DMEM containing $5 \%$ fetal calf serum for $30 \mathrm{~min}(10)$. Cells were treated with vehicle, clioquinol alone $(7 \mu \mathrm{g} / \mathrm{ml})$, zinc alone $(50 \mu \mathrm{M}$ $\mathrm{ZnCl}_{2}$ ), and clioquinol-zinc $(7 \mu \mathrm{g} / \mathrm{ml}$ clioqui$\mathrm{nol} / 50 \mu \mathrm{M} \mathrm{ZnCl}_{2}$ ) at $37^{\circ} \mathrm{C}$ for 30,60 , and $90 \mathrm{~min}$. At the end of incubation, chambers were mounted on the microscope stage of an inverted

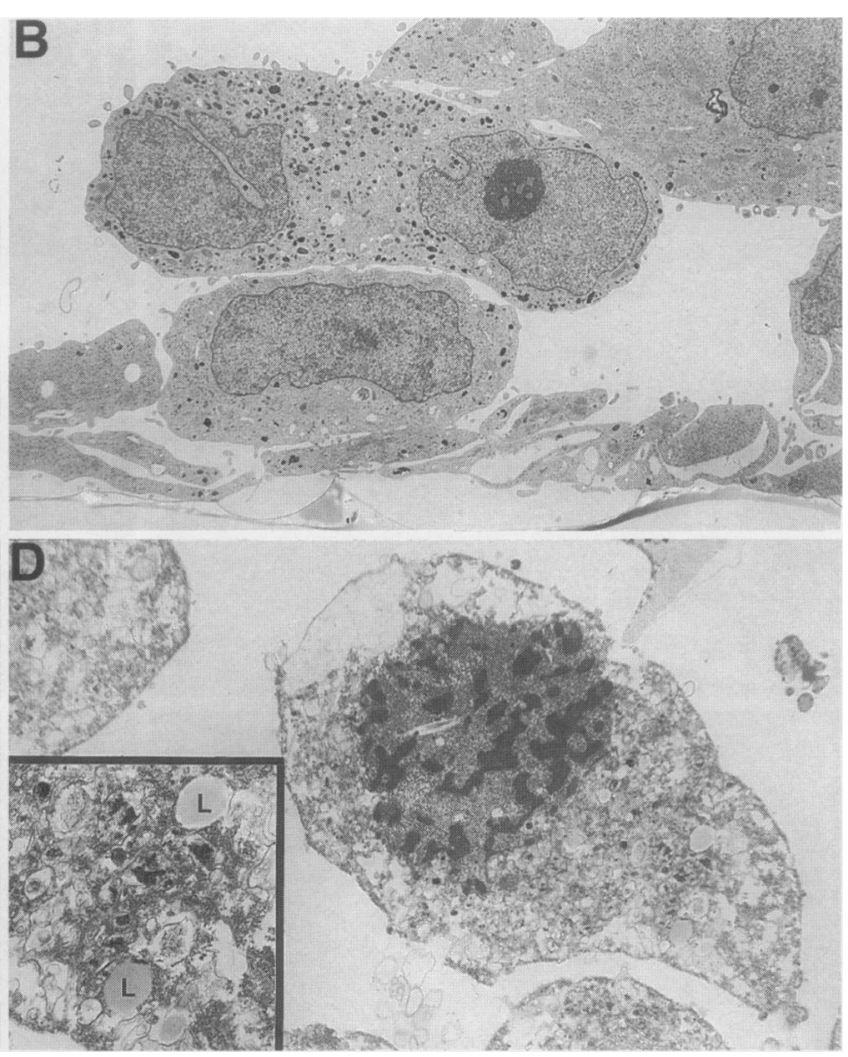

the upper inset $(\times 15,000)$ demonstrate focal adhesion plaques, and the lower inset $(\times 14,300)$ shows increased melanosomes seen as a result of clioquinol treatment. (D) Melanoma cells following treatment with $7.5 \mu \mathrm{g} / \mathrm{ml}$ clioquinol in combination with 150 $\mu \mathrm{M}$ zinc chloride $(\times 7200)$. The inset $(\times 15,600)$ demonstrates damaged mitochondria; L, lipid droplets seen in necrotic cells.

confocal laser scanning microscope (LSM410, Zeiss, Gottingen, Germany) equipped with an external argon-krypton laser. Images from representative fields of the different treatment conditions were taken under identical contrast and brightness settings to allow for semiquantitative comparison of the mitochondrial fluorescence intensities. Images were printed with a Fujix Pictrography 3000 color printer (Fujifilm, Tokyo, Japan) using Adobe Photoshop software (Adobe Systemis, Mountain View, CA).

\section{Statistics}

Significant differences between two groups were determined using an unpaired, two-tailed Student's $t$ test. Results are expressed as the mean \pm SEM. 

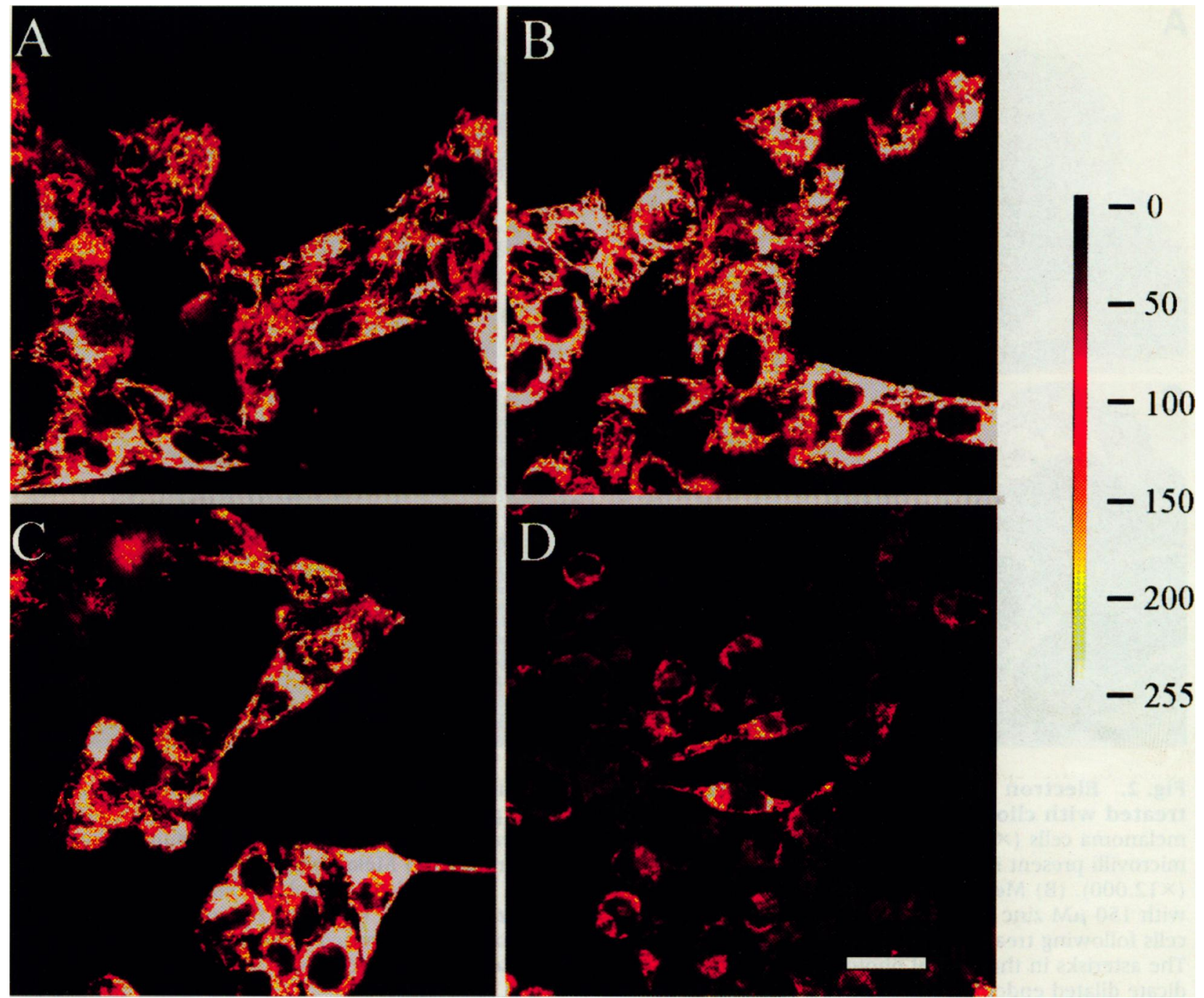

Fig. 3. Microscopic analysis of mitochondrial membrane potential in MMAN cells. Cells were loaded with the mitochondrial potential-sensitive dye MitoTracker Red CMXRos and incubated with vehicle alone (DMSO) (A), $50 \mu \mathrm{M} \mathrm{ZnCl}_{2}$ (B), clioquinol $(7 \mu \mathrm{g} / \mathrm{ml})(\mathrm{C})$, or clioquinol $/ \mathrm{ZnCl}_{2}(7 \mu \mathrm{g} / \mathrm{ml}$

\section{Results}

Clioquinol-ion chelates were formed with ions common in the intestine. These ions include zinc, calcium, magnesium, and copper. Only clioquinol-zinc chelate demonstrated appreciable cytotoxicity to melanoma cells, and this toxicity appeared to be at least additive, as it occurred at a concentration at which zinc alone or clioquinol alone had little cytotoxicity (Fig. 1). Chelates of clioquinol with calcium, magnesium, or copper did not cause appreciable cytotoxicity, nor did zinc in the presence of other chelators such as penicillamine or 1,10 phenanthroline clioquinol/50 $\mu \mathrm{M} \mathrm{ZnCl}_{2}$ ) (D) for $90 \mathrm{~min}$. Images of representative fields were recorded under identical conditions. Color bar displays gray levels (relative fluorescence intensities) that correspond to colors. Bar, $25 \mu \mathrm{m}$. (data not shown). The cytotoxicity observed by treatment with clioquinol-zinc chelate was irreversible when the melanoma cells were replated in the absence of chelate.

In order to further study the morphologic changes induced by clioquinol, zinc, and the combination of both compounds, electron microscopy of MMAN cells was performed. The combination of zinc and clioquinol caused mitochondrial swelling, an increase in melanosomes, lipid droplet accumulation, hydropic vacuolization, and nuclear changes consistent with necrosis (Fig. 2). This cytotoxicity took place at doses 
of clioquinol and zinc which by themselves were not cytotoxic, implying at least additive toxicity.

The rapid onset of action of clioquinol-zinc chelate and the changes visible on electron microscopy suggested that the target of this chelate might be the mitochondria. In order to confirm this, melanoma cells were incubated in the presence of a mitochondrial potential-dependent dye. Cells incubated in the presence of clioquinol-zinc chelate showed nearly complete loss of mitochondrial potential after $90 \mathrm{~min}$ of incubation (Fig. 3). Cells incubated in the presence of vehicle alone, clioquinol alone, or zinc alone showed no loss of mitochondrial potential (Fig. 3).

\section{Discussion}

8-hydroxyquinolines are compounds that have had wide oral and topical use. Oral clioquinol has been used as an antiparasitic agent and for the treatment of acrodermatitis enteropathica, a genetic disorder of zinc absorption (1-6). Oral use of this agent has diminished in recent years because of the epidemiologic association of oral clioquinol with subacute myelo-optic neuropathy (SMON), a neuropathy associated with paresthesias and gait disturbances, as well as retinal toxicity (1-6). Despite intensive study, the mechanism of how clioquinol causes neurotoxicity has not been elucidated.

In order to cause neurotoxicity, clioquinol must be converted to a lipophilic compound that can be orally absorbed and can enter neuronal tissue. Metabolism is an unlikely pathway as clioquinol is metabolized to its glucuronide and sulfate, which is not lipophilic and is readily excreted $(11,12)$. The compound must also demonstrate greater toxicity to intact cells than clioquinol itself. Magnesium-clioquinol chelate has been reported to cause mitochondrial uncoupling in cell-free systems $(13,14)$, but we observed no cytotoxicity of magnesium-clioquinol chelate in intact cells. Finally, the clioquinol derived toxin must be present in humans. The chelate of clioquinol with zinc ions fulfills all of these criteria. Clioquinol-zinc chelate shows rapid cytotoxicity to human cells and is lipophilic. Zinc ions have been shown to alter the conformation and to inhibit the biologic activity of neuropeptides related to neuronal survival, including nerve growth factor (15). The combination of mitochondrial toxicity observed in this study and the inhibition of nerve growth factor activity seen in the presence of zinc makes zinc a likely culprit in SMON. Clioquinol-zinc chelate has been demonstrated to be present in humans by virtue of correction of the zinc deficit in acrodermatitis enteropathica, and it has been demonstrated to facilitate uptake and distribution of zinc to neuronal tissues $(11,16)$. This is the first report showing that clioquinol-zinc chelate, which was of benefit in the treatment of zinc deficiency, is also a potent mitochondrial toxin. Thus, clioquinol-zinc chelate fulfills all of the criteria of a cause for SMON.

\section{Acknowledgments}

J. L. A. was supported by grants from the Dermatology Foundation, Howard Hughes Medical Institute, the Thomas B. Fitzpatrick Research Award from the KAO Corporation, and grant RO3AR44947 from the National Institutes of Health.

\section{References}

1. Tsubaki T, Honma Y, Hoshi M. (1971) Neurological syndrome associated with clioquinol. Lancet $\mathbf{1}$ : 696-697.

2. Oakley GP. (1973) The neurotoxicity of the halogenated hydroxyquinolines. JAMA 225: 395-397.

3. Nakae K, Yamamoto S, Igata A. (1971) Subacute myelo-optico-neuropathy (SMON) in Japan. A community survey. Lancet 23: 510-512.

4. Sturtevant FM. (1980) Zinc deficiency, acrodermatitis enteropathica, optic atrophy, subacute myelo-optic neuropathy and 5,7-dihalo-8quinolinols. Pediatrics 65: 610-613.

5. Moynahan EJ. (1974) Acrodermatitis enteropathica: a lethal inherited zinc deficiency disorder. Lancet 2: 399-400.

6. Toyokura Y, Takasu T. (1975) Clinical features of SMON. Jpn. J. Med. Sci. Biol. 28S: 87-99.

7. Hanakago R, Uono M. (1981) Clioquinol intoxication occurring in the treatment of acrodermatitis enteropathica with reference to SMON outside of Japan. Clin. Toxicol. 18: 1427-1434.

8. Vink J, Thomas L, Etoh T, Bruijn JA, Mihm MC Jr, Gattoni-Celli S, Byers HR. (1993) Role of beta-1 integrins in organ specific adhesion of melanoma cells in vitro. Lab. Invest. 68: 192-203.

9. Rossouw DJ, Cinti S, Dickersin GR. (1986) Liposarcoma: an ultrastructural study of 15 cases. Am. J. Clin. Pathol. 85: 649-667.

10. Poot M, Zhang YZ, Kramer JA, et al. (1996) Analysis of mitochondrial morphology and function with novel fixable fluorescent stains. J. Histochem. Cytochem. 44: 1363-1372.

11. Weismann K, Knudsen L. (1978) Effects of penicillamine and hydroxyquinoline on absorption of 
orally ingested 65zinc in the rat. J. Invest. Dermatol. 71: $242-244$.

12. Ohshima N, Kotaki H, Saitoh Y, Nakagawa F, Tamura Z. (1989) Sex difference of the metabolic disposition of clioquinol in rats. J. Pharmacobiodyn. 12: 371-377.

13. Inouye B, Ogata M. (1979) Effect of chinoform on the function of biological membranes. Physiol. Chem. Phys. 11: 49-57.

14. Hagihara M, Yagi K. (1975) Effect of albumin on uncoupling of oxidative phosphorylation by chinoform in rat liver mitochondria. Experientia 31: 1069-1070.

15. Ross GM, Shamovsky IL, Lawrance G, et al. (1997) Zinc alters conformation and inhibits biological activities of nerve growth factor and related neurotropins. Nat. Med. 3: 872-878.

16. Tjalve H, Stahl K. (1984) Effect of 5-chloro-7iodo-8-hydroxyquinoline on the uptake and distribution of nickel, zinc, and mercury in mice. Acta Pharmacol. Toxicol. 55: 65-72. 